\title{
Beschäftigungsförderung Jugendlicher unter der ÖVP-FPÖ-Regierung
}

\begin{abstract}
Die österreichische Jugendberufshilfe zwischen wirtschaftskonservativer Arbeitsmarktintegration und rechtspopulistischer Diskriminierung
\end{abstract}

In dem Wandel der Beschäftigungsförderung Jugendlicher spiegeln sich arbeits-, bildungs- und sozialpolitische wie auch demographische Entwicklungen wider. Während sich in Österreich der Zugang zu einer Lehrstelle bis Mitte der 90er Jahre wegen eines Überschusses an Lehrstellen unproblematisch darstellte, werden seitdem die Unterstützungsleistungen systematisch ausgebaut. Zu Beginn der letzten rot-schwarzen Regierungsphase (2007 bis 2017) wurde sogar eine Ausbildungsgarantie eingeführt, die Jugendlichen, die keine Lehrstelle finden, eine Ausbildung in einer sogenannten „überbetrieblichen Lehre” ermöglicht. Diese Lehren werden entweder in Lehrwerkstätten durchgeführt oder durch eine Kombination von Unterricht und Betriebspraktika organisiert.

$\mathrm{D}$ ie Ausbildungsgarantie, die als Youth Guarantee Vorbild für die europäische Ausbildungsförderung wurde, steht neben der überbetrieblichen

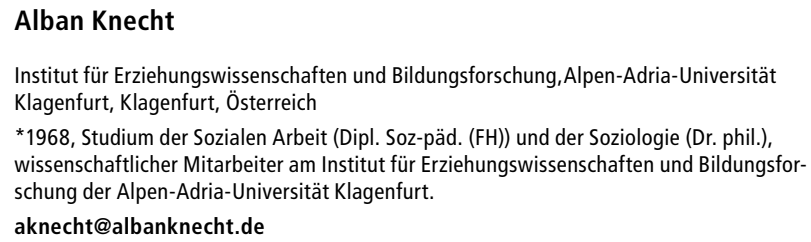

\section{Michael Bodenstein}

Wien, Österreich

*1981, Studium der Komparatistik und Germanistik, seit 2012 Mitarbeiter bei PROSA („Projekt Schule für alle")

michael.bodenstein@vielmehr.at

Zusammenfassung Die Autoren betrachten die Veränderungen in der Beschäftigungsförderung Jugendlicher durch die seit 2017 amtierende österreichische Bundesregierung. Nach einem Einblick in die Ausgangssituation wird die beschäftigungspolitische Agenda der Koalition aus ÖVP und FPÖ anhand der Eingriffe in soziale Rechte und Leistungen bei österreichischen Lehrlingen sowie minderjährigen Aslybewerber_innen illustriert. Hierbei zeigt sich, dass jenseits vermeintlicher ideologischer Reibungsflächen innerhalb der Koalition oder vordergründiger Symbolpolitiken das Konzept der Dualisierung zu einem tieferen Verständnis der Regierungsarbeit beitragen kann.

Schlüsselwörter Beschäftigungspolitik Jugendlicher, Jugendliche Asylsuchende, Österreich, Jugendberufshilfe, Dualisierung
Lehre auf zwei weiteren Säulen: Die am dänischen Modell orientierte Produktionsschule bietet Basisbildung im Rahmen von praxisorientiertem Lernen, das mit einer Betreuung durch Pädagog_innen verschränkt ist. Das Jugendcoaching stellt ein bislang niederschwelliges Beratungsangebot für Jugendliche dar, die Probleme mit der Schule oder beim Übergang in die Lehre haben. $\mathrm{Zu}$ den Jugendlichen wird dazu über die Schule oder die offene Jugendarbeit Kontakt aufgebaut und in einem Zeitraum bis zu einem Jahr beraten.

Der starke Ausbau und die umfangreiche finanzielle Förderung folgen dem auch von der EU unterstützen Leitbild des Sozialinvestitionsstaates. Der Ansatz der Sozialinvestitionen verspricht eine Versöhnung von wohlfahrtsstaatlichen Aktivitäten und Anforderungen des produktiven und wettbewerbsfähigen Staates, indem die Renditen von sozialen Leistungen in den Vordergrund gerückt werden. Diese Politik, die v. a. in Großbritannien als Antwort auf Thatchers neoliberale Politik formuliert wurde und durch das Schröder-BlairPaper in den deutschsprachigen Raum gekommen ist, setzt einen Schwerpunkt auf „Humankapitalbildung“, auf Forschung und Entwicklung, aber auch auf frühkindliche Bildung (Hemerijck 2013) zu Lasten von sogenannten unproduktiven Bereichen wie Pensionen oder Leistungen der Mindestsicherung.

Untersuchungen zeigen, dass mit der Umsetzung des Investitionsstaats zunehmend - im Sinne der Absicherung der Renditen - auf aktivierende Elemente zurückgegriffen wird (Atzmüller und Knecht 2016; Knecht 
und Atzmüller 2017). Dementsprechend wurde 2017, am Ende der rot-schwarzen Regierungsperiode, die Ausbildungsgarantie durch eine Ausbildungspflicht ersetzt, die - im Anschluss an die Schulpflicht - Jugendliche zwischen 15 und 18 dazu zwingt, einer Ausbildung in Form eines Schulbesuchs oder einer Lehre nachzugehen. Das bisher als freiwilliges und niederschwelliges Angebot gepriesene Jugendcoaching kommt nun in den Sog der Verpflichtung. Das wird mit einer Veränderung seines Charakters und der Arbeit der Coaches einhergehen, da Niederschwelligkeit und Pflicht Prinzipien sind, die einander ausschließen (Knecht 2016; Mayrhofer 2012). Ein umfangreiches Monitoringsystem („Monitoring Ausbildung bis 18 - MAB“), das die Grundlage für den Vollzug der Ausbildungspflicht und der Bestrafung schafft, wurden bereits installiert.

\section{Die Agenda der ÖVP-FPÖ-Regierung}

Nach nur einem Jahr Regierungszeit der ÖVP-FPÖKoalition haben sich im Bereich der Lehre bereits einige Änderungen ergeben. So hat die neue Regierung den Jugendvertrauensrat abgeschafft. Er hatte für minderjährige Angestellte eine ähnliche Funktion wie der Betriebsrat für Erwachsene und diente der Einübung von Mitbestimmung in der Praxis. Erst im Jahre 2013 waren auch in der überbetrieblichen Lehre Vertrauensräte installiert worden. Die neue Regierung hat nun den Jugendvertrauensrat zugunsten einer Absenkung des Wahlalters für Betriebsräte auf 16 Jahre gestrichen. Allerdings ist ein Drittel aller Lehrlinge (sie werden in Österreich noch so genannt) jünger als 16 Jahre und verliert somit vollends die Möglichkeit der betrieblichen Mitbestimmung. Volljährige Lehrlinge sind überdies von den neuen Arbeitszeitregelungen betroffen, die es ermöglichen, Arbeitnehmer_innen bis zu zwölf Stunden am Tag arbeiten zu lassen (statt wie bisher zehn).

Darüber hinaus wurde die Beihilfe, die Lehrlinge in der überbetrieblichen Ausbildung als Lohn erhalten, um circa die Hälfte auf $325 €$ gesenkt.

Aus Sicht der Regierung sprachen verschiedene Gründe für die Kürzung: Wirtschaftsnahe Kreise haben die überbetriebliche Ausbildung immer kritisiert, weil sich - trotz des allgemeinen Mangels an Lehrstellen -, nicht für alle offenen Stellen Bewerber_innen finden lassen. Insbesondere unattraktive Lehrstellen in der Gastronomie und im Tourismus lassen sich manchmal nicht besetzen. Für die FPÖ mag eine Rolle spielen, dass unter den Teilnehmer_innen der überbetrieblichen Ausbildung auch Jugendliche mit Migrationserfahrung sind. Diese Jugendlichen haben des öfteren Schwierigkeiten bei der Lehrstellensuche, da Vorurteile bestehen und sie diskriminiert werden (vgl. Imdorf 2017). Solche Erfah- rungen wurden auch in Interviews mit Jugendcoaches berichtet, die bezüglich der Vermittlung von Lehrstellen mit Betrieben in Kontakt waren (AG Jugendcoaching 2018). Die FPÖ favorisiert den weiteren Ausbau der bereits existierenden finanziellen Lehrstellenförderungen für Unternehmen, die Lehrlinge aufnehmen. Insgesamt möchte die neue Regierung auch mehr Druck auf Jugendliche aufbauen, reguläre Lehrstellen anzunehmen: Lehrlinge der überbetrieblichen Lehre sollen verpflichtet werden, mittels Bewerbungen permanent zu versuchen, auf reguläre Lehrstellen zu wechseln (Szigetvari 2018). Betrachtet man die Pläne der ÖVP-FPÖ-Regierung, so zeigt sich ein wirtschaftsfreundlich-paternalistischer Politikstil: Die Politik verfolgt scheinbar eine neoliberale Sparpolitik, die den Abbau von partizipativer Mitbestimmung und den Abbau von Sozialleistungen umfasst. Sie plädiert allerdings gleichzeitig für ein teures System der Subvention von Unternehmen, die Lehrlinge aufnehmen - und entlastet Eltern durch einen teuren steuersenkenden Kinderabsetzbetrag, der allerdings in erster Linie besserverdienenden Familien zugutekommt.

\section{Arbeitsmöglichkeiten minderjähriger Asyl- bewerber werden abgeschafft}

Asylsuchenden Jugendlichen war es bisher während des Verfahrens einzig in eng definierten Bereichen möglich zu arbeiten. Die restriktive Politik gestand ihnen lediglich Arbeitsbereiche wie Ernte- oder Saisonarbeit sowie gemeinnützige Tätigkeiten zu. Gemeinnützige Arbeiten müssen im Vorfeld behördenseitig akzeptiert und den Flüchtlingsunterkünften zur Kenntnis gebracht werden, da im schlimmsten Fall der Verlust der Unterkunft droht. Dies zeugt von einer Überbürokratisierung dieses Bereiches, welche als verlängerter Arm der exkludierenden Politik fungiert. Solche Barrieren bestanden auch, wenn ein jugendlicher Asylsuchender einer Lehre während des laufenden Asylverfahrens aufnehmen wollte. Lehren waren nur in Mangelberufen nach Zustimmung der in Österreich „das Arbeitsmarktservice“ (AMS) genannten Arbeitsverwaltung gestattet. Das AMS durfte nicht aktiv bei der Lehrstellensuche helfen, musste aber prüfen, ob es vorzuziehende österreichische Bewerber_innen gibt und ob die Erklärung des Arbeitsgebers/der Arbeitsgeberin, dass keine andere Person diese Tätigkeit ausüben will und kann, gegeben ist. Gleichzeitig erfolgt mit dem Vollenden des Pflichtschulalters, also ca. mit 15, der Ausschluss aus der Ausbildungsgarantie und nun auch aus der Ausbildungsverpflichtung. Es folgt der zynischen Logik der Regierung, Integrations- und Bildungsprojekte, die sich an diese Altersgruppe richten, zusätzlich durch massive Kürzungen beim AMS zu gefährden. So sind von der 
Streichung der Teilfinanzierung durch das AMS viele CORE-Projekte wie das Jugendcollege, die Geflüchteten lerner_innenzentrierte Basisbildung vermittelt, betroffen (Hagen 2018). Jugendliche werden somit auch hinsichtlich sinnvoller Bildungsangebote zur Passivität verdammt und sind darauf verwiesen zu warten, bis das Verfahren entschieden ist, was mitunter dauern kann (Bodenstein und Knecht 2017).

Im September 2018 wurde die Möglichkeit von Asylwerber_innen, eine Ausbildung in einem Mangelberuf zu machen, abgeschafft. Vor dieser Änderung entstand eine koalitionsinterne Diskussion darüber, ob es erwünscht ist, dass jugendliche Asylsuchende im Verfahren eine Ausbildung machen dürfen. Die Wirtschaftskammern und selbst Teile der Regierungspartei ÖVP stellten sich in dieser Frage gegen die Regierung (Die Presse 2018). Hier stießen die Leitbilder von verschiedenen Teilen der Regierung aufeinander und - ähnlich wie bei den Diskussionen zur Einführung des 12-Stunden-Arbeitstages - wurde die Reibungsfläche zwischen einem wirtschaftsfreundlich-neoliberalen Kurs und der populistischen Ausgrenzungspolitik der FPÖ ersichtlich, die sich gerne als „soziale Heimatpartei“ darstellt. Dass es nicht wirklich darum geht, Lehrstellen für österreichische Jugendliche gegenüber der asylsuchenden Konkurrenz zu schützen, zeigt der geringe Anteil von Asylbewerbern in der Lehre - nur 1000 von 106.000 Lehrlingen sind asylsuchend. Häufig sind die Lehren in den Mangelberufen nicht sonderlich attraktiv und können daher nicht besetzt werden. Bei der Abschaffung der Lehre geht es für die Politik in erster Linie darum, sich durch eine symbolische Politik der Ausgrenzung zu positionieren, auf Kosten sinnvoller Jugendpolitik.

\section{Dualisierung als Programmatik}

Ähnlich wie in einigen anderen Ländern wird unter dem Deckmantel einer Anti-Asyl-Politik eine neoliberale Politik des radikalen Abbaus an sozialen Leistungen und Rechten vorangetrieben, der den Jugendbereich genauso trifft wie die Bereiche Mindestsicherung, Arbeitszeitregelung (mit der Einführung der täglichen Höchstarbeitszeit von zwölf Stunden) oder den zweiten Arbeitsmarkt (Wegfall der „Aktion 20.000“, die 20.000 Arbeitsplätze für über 50jährige schaffte), allerdings neue Vorteile, insbesondere für die industrielle Wirtschaft und Besserverdienende, schafft. Aber nicht nur hier geht die Schere zwischen oben und unten weiter auf.

Um das populistische Pferd reiten zu können, werden die schon bisher auch im internationalen Vergleich (OECD 2016) bescheidene Rechte asylsuchender Jugendlicher weiter beschnitten: „Unserer Jugend sollen alle Chancen offenstehen. Unsere Kinder sollen zu mündigen, eigenverantwortlichen Bürgerinnen und Bürgern heranwachsen können. Ziel ist die Förderung ihrer Entwicklung als eigenständige Persönlichkeiten, um ein selbständiges Leben führen und ihre Potenziale und Talente optimal nutzen und entfalten zu können. Überaus wichtig ist, dass Jugendliche eine positive Zukunftsperspektive haben. Hier muss die Politik alles daransetzen, um dies zu gewährleisten.“

„Unserer Jugend“ und „Unsere Kinder“ muss hier als Ankündigung einer diskriminierenden, ausgrenzenden Kinder- und Jugendpolitik gelesen werden. Auch hier geht die Schere auf, nämlich zwischen österreichischen Kindern und Jugendlichen, denen hier scheinbar Privilegien zugestanden werden und benachteiligten asylsuchenden Kindern und Jugendlichen.

Die Änderungen der Beschäftigungspolitik für Jugendliche können mit dem Konzept der Dualisierung besser verstanden werden. Es beschreibt eine Tendenz neuer (neoliberaler) Politik unterschiedlichen Bevölkerungsgruppen unterschiedliche Pflichten und Rechte zuzuteilen (Emmenegger et al. 2012; Dallinger und Fückel 2014). Auch wenn die Ausgrenzungspolitik der Regierung sich einer populistischen Symbolpolitik bedient, die den Anschein erweckt, sich alleine gegen Asylsuchende zu wenden, ist sie de facto in mehrfacher Hinsicht dualisierend: Denen, die in der Gesellschaft gut positioniert sind, werden mehr Rechte zugeteilt, während Benachteiligte in ihren sozialen Rechte beschnitten und ihnen gleichzeitig mehr Pflichten auferlegt werden. Dieses „Spiel“, das sich in vielen Politikbereichen zeigt, wird sowohl für die allgemeine Beschäftigungsförderung von Jugendlichen wiederholt als auch - durch das Lehrstellenverbot - für die asylsuchenden Jugendlichen. Es ist genau diese Art von dualisierender Politik, die sich auch in der zynischen „Lösung“ des Konfliktes zwischen den wirtschaftsliberalen Kräften, die gerne mehr Lehrlinge zur Verfügung hätten, um alle (auch unattraktive) Lehrstellen besetzen zu können und den „Hardlinern “ einer Anti-Asyl-Politik zeigt: Die Koalition unterbindet zwar, dass die bisher tätigen asylsuchende Lehrlinge ihre Lehre beenden können und möchte insbesondere verhindern, dass eine Lehre einer Abschiebung im Weg stehen könnte. Allerdings zeichnet sich ab, dass die rot-weiß-rot Karte, die der deutschen Green-Card entspricht, für Lehrlinge geöffnet wird. Das würde bedeuten, dass Jugendliche aus Nicht-EU-Ländern sich um eine Lehre in Österreich bewerben können. Die Regierung macht somit eine neue Unterscheidung auf: Zwischen asylsuchenden Jugendlichen aus Fluchtländern, die keine Lehre mehr machen dürfen und Jugendlichen aus sicheren Ländern, die sich um einen rot-weiß-rot Lehrlingskarte bewerben dürfen. Gemäß der dualisie- 
renden Politik müssen natürlich auch die Rechte der rot-weiß-rot-Lehrlinge begrenzt bleiben: Die Rot-weißrot-Karte schließt den Zugang zu Sozialleistungen wie der Mindestsicherung kategorisch aus. Vielleicht gibt es nur zwei verlässliche Prinzipien der neuen Regierung: „Teile und herrsche“, und „Trete nach unten“.

Funding. Open access funding provided by University of Klagenfurt.

Open Access. Dieser Artikel wird unter der Creative Commons Namensnennung 4.0 International Lizenz (http://creativecommons.org/ licenses/by/4.0/deed.de) veröffentlicht, welche die Nutzung, Vervielfältigung, Bearbeitung, Verbreitung und Wiedergabe in jeglichem Medium und Format erlaubt, sofern Sie den/die ursprünglichen Autor(en) und die Quelle ordnungsgemäß nennen, einen Link zur Creative Commons Lizenz beifügen und angeben, ob Änderungen vorgenommen wurden.

\section{Literatur}

Atzmüller, R., \& Knecht, A. (2016). Neoliberale Transformation der österreichischen Beschäftigungspolitik für Jugendliche. SWS-Rundschau, 56(1), 112-132.

Bodenstein, M., \& Knecht, A. (2017). Existentielles Warten. Bildungswege und Alltagsstrategien jugendlicher Asylsuchender. Asyl Aktuell. Zeitschrift der Asylkoordination Osterreich, 16(4), 25-27.

Dallinger, U., \& Fückel, S. (2014). Politische Grundlagen und Folgen von Dualisierungsprozessen: Eine politische Ökonomie der Hartz-Reformen. WSI Mitteilungen, 72(3), 182-191.

Die Presse (2018). Immer mehr ÖVP-Vertreter gegen Abschiebung von Lehrlingen. In: Die Presse, vom 24.08.2018. https://diepresse.com/home/innenpolitik/5485130/Immer-mehr-OeVPVertreter-gegen-Abschiebung-vonLehrlingen. Zugegriffen: 14. Okt. 2018.

Emmenegger, P., et al. (Hrsg.). (2012). The age of dualization. The changing face of Inequlity in deindustrializing societies. Oxford: University Press.

Hagen, L. (2018). AMS-Kürzungen - Armutszeugnis und Zukunftsraub. In: Der Standard, 28.02. https://derstandard.at/2000075198768/AMS-Kuerzungen-Armutszeugnis-und-Zukunftsraub. Zugegriffen: 12. Okt. 2018.

Hemerijck, A. (2013). Changing welfare states. Oxford: Oxford University Press.

Imdorf, C. (2017). Diskriminierung in der beruflichen Bildung. In A. Scherr \& al (Hrsg.), Handbuch Diskriminierung. Wiesbaden: Springer.

Jugendcoaching, A. G. (2018). Jugendcoaching - Ein Beratungsangebot der Beschäftigungsförderung für benachteiligte Jugendliche in Österreich. Linz: JKU. Unveröffentlichter Forschungsbericht des Seminars ,Spezialisierung im Praxisfeld', WS 2017/18

Knecht, A. (2016). Wenn soziale Dienstleistungen nicht in Anspruch genommen werden. Das österreichische Beratungsangebot Jugendcoaching zwischen Niederschwelligkeit und Verpflichtung. Soziale Arbeit, 65(1), 17-22.

Knecht, A., \& Atzmüller, R. (2017). Von der Ausbildungsgarantie zur Ausbildungspflicht. Die Entwicklung der österreichischen Beschäftigungspolitik für Jugendliche. Neue Praxis, 47(3), 239-252.

Mayrhofer, H. (2012). Niederschwelligkeit in der Sozialen Arbeit: Funktionen und Formen aus soziologischer Perspektive. Wiesbaden: Springer.

OECD (2016). Making Integration Work. Refugees and Others in Need of Protection. OECD Publishing, Paris. http://dx.doi. org/10.1787/9789264251236-en. Zugegriffen: 14. Okt. 2018.

Szigetvari, András (2018). Mehr Geld und Imagepolitur für die Lehre. In: Der Standard, 18. Sept., S. 15.

Hier steht eine Anzeige.

黛 Springer 